\title{
Results of the initial in-brace correction-effect
}

\author{
Kay Steffan ${ }^{1 *}$, Hans-Jörg Heinen ${ }^{2}$ \\ From 11th International Conference on Conservative Management of Spinal Deformities - SOSORT 2014 \\ Annual Meeting \\ Wiesbaden, Germany. 8-10 May 2014
}

\section{Background}

Brace treatment for scoliosis is effective. The short term in-brace correction of the Cobb angle is associated with end results and success of bracing. Irrespective of the factors which determine the correction the measurement of the Cobb angle is a possibility to evaluate the brace quality.

We have investigated the braces from one orthotist over a fixed period of time and have described the connection between age, initial Cobb angle and the achieved correction effect.

\section{Methods}

Study design: retrospective. Population: n713 patients diagnosed as having juvenile and adolescent idopathic scoliosis. We differentiated between 1085 curvatures and assessed the changes. Required was a complete ap$y$-ray film without brace not older than 6 months prior to the start of the brace treatment and the second $\mathrm{x}$-ray film 2 month after the brace fitting.

The correction effect was described by the difference between the Cobb angle of the $2 \mathrm{x}$-ray films and expressed as a percentage. Groups were matched regarding Cobb angle and age.

\section{Results}

Median reported correction effect for all 1085 curvatures was $65.4 \%$.

Patients with $\mathrm{Cobb}<25^{\circ}$ the mean correction was $68.91 \%$, Cobb $26^{\circ}$ to $40^{\circ}$ mean correction $38.9 \%$, Cobb > $41^{\circ}$ correction $28.42 \%$.

\section{Age groups}

$<10 y$ mean effect $87.56 \%$, N. 57

Cobb $<25^{\circ} 95.97 \%$, Cobb $26^{\circ}$ to $40^{\circ} 63.12 \%$, Cobb > $40^{\circ} 34.72 \%$

\footnotetext{
${ }^{1}$ Asklepios Katharina Schroth Klinik, Bad Sobernheim, Germany

Full list of author information is available at the end of the article
}

$10-12 y r$ mean effect $58.83 \%$, N. 100

Cobb $<25^{\circ} 74.95 \%$, Cobb $26^{\circ}$ to $40^{\circ} 41.79 \%$, Cobb > $40^{\circ} 31.67 \%$

$13-14$ yr mean effect 50,2\%, N. 238

Cobb $<25^{\circ} 67.25 \%$, Cobb $26^{\circ}$ to $40^{\circ} 38.91 \%$, Cobb > $40^{\circ} 28.69 \%$

$>14 y$ mean effect $42.17 \%$, N. 318

Cobb $>25^{\circ} 57.47 \%$, Cobb $26^{\circ}$ to $40^{\circ} 36.58 \%$, Cobb > $40^{\circ} 27.78 \%$

\section{Conclusions}

The achieved corrections show what we have already expected that younger and less matured patients have the best results. As maturity and the Cobb angle increase the correction effect was reduced by the more structural components of the curvatures.

However the mean correction effect of $65.4 \%$ seems to be encouraging. For us the results make clear that to start with bracing early even with Cobb angles below $25^{\circ}$ seems to be a sufficient decision within the conservative scoliosis treatment.

Authors' details

${ }^{1}$ Asklepios Katharina Schroth Klinik, Bad Sobernheim, Germany. ${ }^{2}$ Sanomed, Bad Sobernheim, Germany.

Published: 4 December 2014

doi:10.1186/1748-7161-9-S1-O32

Cite this article as: Steffan and Heinen: Results of the initial in-brace correction-effect. Scoliosis 2014 9(Suppl 1):032. 